\title{
Fixed point theorems for $N$-generalized hybrid mappings in uniformly convex metric spaces
}

\section{Withun Phuengrattana*}

\section{"Correspondence:}

withun_ph@yahoo.com

Mathematics Program, Faculty of

Science and Technology, Nakhon

Pathom Rajabhat University,

Nakhon Pathom, 73000, Thailand

\begin{abstract}
In this paper, we prove some fixed point theorems for $\mathrm{N}$-generalized hybrid mappings in both uniformly convex metric spaces and CAT(0) spaces. We also introduce a new iteration method for approximating a fixed point of $\mathrm{N}$-generalized hybrid mappings in CAT(0) spaces and obtain $\Delta$-convergence to a fixed point of $\mathrm{N}$-generalized hybrid mappings in such spaces. Our results improve and extend the corresponding results existing in the literature.
\end{abstract}

MSC: $47 \mathrm{H} 09 ; 47 \mathrm{H} 10$

Keywords: fixed point; uniformly convex metric spaces; CAT(0) spaces; generalized hybrid mappings

\section{Introduction and preliminaries}

Let $C$ be a nonempty closed subset of a metric space $(X, d)$ and let $T$ be a mapping of $C$ into itself. The set of all fixed points of $T$ is denoted by $F(T)=\{x \in C: x=T x\}$. In 1970, Takahashi [1] introduced the concept of convex metric spaces by using the convex structure as follows.

Definition 1.1 Let $(X, d)$ be a metric space. A mapping $W: X \times X \times[0,1] \rightarrow X$ is said to be a convex structure on $X$ if for each $x, y \in X$ and $\lambda \in[0,1]$,

$$
d(z, W(x, y, \lambda)) \leq \lambda d(z, x)+(1-\lambda) d(z, y)
$$

for all $z \in X$. A metric space $(X, d)$ together with a convex structure $W$ is called a convex metric space which will be denoted by $(X, d, W)$.

A nonempty subset $C$ of $X$ is said to be convex if $W(x, y, \lambda) \in C$ for all $x, y \in C$ and $\lambda \in$ $[0,1]$. Clearly, a normed space and each of its convex subsets are convex metric spaces, but the converse does not hold. For each $x, y \in X$ and $\lambda \in[0,1]$, it is known that a convex metric space has the following properties [1, 2]:

(i) $W(x, x, \lambda)=x, W(x, y, 0)=y$ and $W(x, y, 1)=x$;

(ii) $d(x, W(x, y, \lambda))=(1-\lambda) d(x, y)$ and $d(y, W(x, y, \lambda))=\lambda d(x, y)$.

In 1996, Shimizu and Takahashi [3] introduced the concept of uniform convexity in convex metric spaces and studied some properties of these spaces. A convex metric space 
$(X, d, W)$ is said to be uniformly convex if for any $\varepsilon>0$, there exists $\delta_{\varepsilon}>0$ such that for all $r>0$ and $x, y, z \in X$ with $d(z, x) \leq r, d(z, y) \leq r$ and $d(x, y) \geq r \varepsilon$ imply that $d\left(z, W\left(x, y, \frac{1}{2}\right)\right) \leq$ $\left(1-\delta_{\varepsilon}\right) r$. Obviously, uniformly convex Banach spaces are uniformly convex metric spaces.

Let $C$ be a nonempty closed and convex subset of a convex metric space $(X, d, W)$ and let $\left\{x_{n}\right\}$ be a bounded sequence in $X$. For $x \in X$, we define a mapping $r\left(\cdot,\left\{x_{n}\right\}\right): X \rightarrow[0, \infty)$ by

$$
r\left(x,\left\{x_{n}\right\}\right)=\limsup _{n \rightarrow \infty} d\left(x, x_{n}\right) .
$$

Clearly, $r\left(\cdot,\left\{x_{n}\right\}\right)$ is a continuous and convex function. The asymptotic radius of $\left\{x_{n}\right\}$ relative to $C$ is given by

$$
r\left(C,\left\{x_{n}\right\}\right)=\inf \left\{r\left(x,\left\{x_{n}\right\}\right): x \in C\right\}
$$

and the asymptotic center of $\left\{x_{n}\right\}$ relative to $C$ is the set

$$
A\left(C,\left\{x_{n}\right\}\right)=\left\{x \in C: r\left(x,\left\{x_{n}\right\}\right)=r\left(C,\left\{x_{n}\right\}\right)\right\} .
$$

It is clear that the asymptotic center $A\left(C,\left\{x_{n}\right\}\right)$ is always closed and convex. It may either be empty or consist of one or many points. The asymptotic center $A\left(C,\left\{x_{n}\right\}\right)$ is singleton for uniformly convex Banach spaces $[4,5]$ or $\mathrm{CAT}(0)$ spaces [6]. The following lemma obtained by Phuengrattana and Suantai [7] is useful for our results.

Lemma 1.2 Let $C$ be a nonempty closed and convex subset of a complete uniformly convex metric space $(X, d, W)$ and let $\left\{x_{n}\right\}$ be a bounded sequence in $X$. Then $A\left(C,\left\{x_{n}\right\}\right)$ is a singleton set.

One of the special spaces of uniformly convex metric spaces is a CAT( 0$)$ space; see [8]. It was noted in [9] that any $\mathrm{CAT}(\kappa)$ space $(\kappa>0)$ is uniformly convex in a certain sense but it is not a $\mathrm{CAT}(0)$ space. Fixed point theory in $\operatorname{CAT}(0)$ spaces was first studied by Kirk $[9,10]$. He showed that every nonexpansive mapping defined on a bounded closed convex subset of a complete CAT( 0 ) space always has a fixed point. Since then, the fixed point theory for single-valued and multivalued mappings in $\mathrm{CAT}(0)$ spaces has been rapidly developed, and many papers have appeared (e.g., see [11-27]).

Let $(X, d)$ be a metric space. A geodesic path joining $x \in X$ to $y \in X$ (or, more briefly, a geodesic from $x$ to $y$ ) is a map $c$ from a closed interval $[0, l] \subset \mathbb{R}$ to $X$ such that $c(0)=x$, $c(l)=y$ and $d\left(c\left(t_{1}\right), c\left(t_{2}\right)\right)=\left|t_{1}-t_{2}\right|$ for all $t_{1}, t_{2} \in[0, l]$. In particular, $c$ is an isometry and $d(x, y)=l$. The image $\alpha$ of $c$ is called a geodesic (or metric) segment joining $x$ and $y$. When it is unique, this geodesic is denoted by $[x, y]$. Write $c(\alpha 0+(1-\alpha) l)=\alpha x \oplus(1-\alpha) y$ for $\alpha \in(0,1)$. The space $(X, d)$ is said to be a geodesic metric space if every two points of $X$ are joined by a geodesic, and $X$ is said to be uniquely geodesic if there is exactly one geodesic joining $x$ and $y$ for each $x, y \in X$. A subset $Y$ of $X$ is said to be convex if $Y$ includes every geodesic segment joining any two of its points.

A geodesic triangle $\triangle\left(x_{1}, x_{2}, x_{3}\right)$ in a geodesic metric space $(X, d)$ consists of three points $x_{1}, x_{2}, x_{3}$ in $X$ (the vertices of $\triangle$ ) and a geodesic segment between each pair of vertices (the edges of $\triangle$ ). A comparison triangle for the geodesic triangle $\triangle\left(x_{1}, x_{2}, x_{3}\right)$ in $(X, d)$ is a 
triangle $\bar{\triangle}\left(x_{1}, x_{2}, x_{3}\right):=\triangle\left(\bar{x}_{1}, \bar{x}_{2}, \bar{x}_{3}\right)$ in the Euclidean plane $\mathbb{E}^{2}$ such that $d_{\mathbb{E}^{2}}\left(\bar{x}_{i}, \bar{x}_{j}\right)=d\left(x_{i}, x_{j}\right)$ for $i, j \in\{1,2,3\}$.

A geodesic metric space is said to be a CAT(0) space if all geodesic triangles satisfy the following comparison axiom: Let $\triangle$ be a geodesic triangle in $X$ and let $\bar{\Delta}$ be a comparison triangle for $\Delta$. Then $\Delta$ is said to satisfy the $\operatorname{CAT}(0)$ inequality if for all $x, y \in \Delta$ and all comparison points $\bar{x}, \bar{y} \in \bar{\Delta}$,

$$
d(x, y) \leq d_{\mathbb{E}^{2}}(\bar{x}, \bar{y}) .
$$

It is well known that any complete, simply connected Riemannian manifold having nonpositive sectional curvature is a CAT( 0$)$ space. Other examples include pre-Hilbert spaces [8], $\mathbb{R}$-trees [16], the complex Hilbert ball with a hyperbolic metric [5], and many others.

If $z, x, y$ are points in a CAT( 0$)$ space and if $m[x, y]$ is the midpoint of the segment $[x, y]$, then the $\operatorname{CAT}(0)$ inequality implies

$$
d(z, m[x, y])^{2} \leq \frac{1}{2} d(z, x)^{2}+\frac{1}{2} d(z, y)^{2}-\frac{1}{4} d(x, y)^{2} .
$$

This is the $(\mathrm{CN})$ inequality of Bruhat and Tits [28], which is equivalent to

$$
d(z, \lambda x \oplus(1-\lambda) y)^{2} \leq \lambda d(z, x)^{2}+(1-\lambda) d(z, y)^{2}-\lambda(1-\lambda) d(x, y)^{2}
$$

for any $\lambda \in[0,1]$. The $\left(\mathrm{CN}^{*}\right)$ inequality has appeared in [29]. Moreover, if $X$ is a $\operatorname{CAT}(0)$ space and $x, y \in X$, then for any $\lambda \in[0,1]$, there exists a unique point $\lambda x \oplus(1-\lambda) y \in[x, y]$ such that

$$
d(z, \lambda x \oplus(1-\lambda) y) \leq \lambda d(z, x)+(1-\lambda) d(z, y)
$$

for any $z \in X$. It follows that $\mathrm{CAT}(0)$ spaces have a convex structure $W(x, y, \lambda)=\lambda x \oplus(1-$ d)y.

\section{Remark 1.3}

(i) By using the $(\mathrm{CN})$ inequality, it is easy to see that $\mathrm{CAT}(0)$ spaces are uniformly convex.

(ii) A geodesic metric space is a $\mathrm{CAT}(0)$ space if and only if it satisfies the $(\mathrm{CN})$ inequality; see [8].

In 2012, Dhompongsa et al. [12] introduced the following notation in CAT(0) spaces: Let $x_{1}, \ldots, x_{N}$ be points in a $\operatorname{CAT}(0)$ space $X$ and $\lambda_{1}, \ldots, \lambda_{N} \in(0,1)$ with $\sum_{i=1}^{N} \lambda_{i}=1$, we write

$$
\bigoplus_{i=1}^{N} \lambda_{i} x_{i}:=\left(1-\lambda_{N}\right)\left(\frac{\lambda_{1}}{1-\lambda_{N}} x_{1} \oplus \frac{\lambda_{2}}{1-\lambda_{N}} x_{2} \oplus \cdots \oplus \frac{\lambda_{N-1}}{1-\lambda_{N}} x_{N-1}\right) \oplus \lambda_{N} x_{N}
$$

The definition of $\bigoplus$ is an ordered one in the sense that it depends on the order of points $x_{1}, \ldots, x_{N}$. Under (1.1) we obtain that

$$
d\left(\bigoplus_{i=1}^{N} \lambda_{i} x_{i}, y\right) \leq \sum_{i=1}^{N} \lambda_{i} d\left(x_{i}, y\right) \quad \text { for each } y \in X .
$$


In 1976, Lim [30] introduced the concept of $\Delta$-convergence in a general metric space. Later in 2008, Kirk and Panyanak [15] extended the concept of Lim to a CAT(0) space.

Definition 1.4 [15] A sequence $\left\{x_{n}\right\}$ in a CAT(0) space $X$ is said to $\Delta$-converge to $x \in X$ if $x$ is the unique asymptotic center of $\left\{u_{n}\right\}$ for every subsequence $\left\{u_{n}\right\}$ of $\left\{x_{n}\right\}$. In this case, we write $\Delta-\lim _{n \rightarrow \infty} x_{n}=x$ and call $x$ the $\Delta$-limit of $\left\{x_{n}\right\}$.

Lemma 1.5 [15] Every bounded sequence in a complete CAT(0) space has a $\Delta$-convergent subsequence.

For any nonempty subset $C$ of a CAT(0) space $X$, let $\pi:=\pi_{C}$ be the nearest point projection mapping from $X$ to a subset $C$ of $X$. In [8], it is known that if $C$ is closed and convex, the mapping $\pi$ is well defined, nonexpansive, and the following inequality holds:

$$
d(x, y)^{2} \geq d(x, \pi x)^{2}+d(\pi x, y)^{2}
$$

for all $x \in X$ and $y \in C$. By using the same argument as in [31, Lemma 3.2], we can prove the following result for nearest point projection mappings in CAT(0) spaces.

Lemma 1.6 Let $C$ be a nonempty closed and convex subset of a complete CAT(0) space $X$, let $\pi: X \rightarrow C$ be the nearest point projection mapping, and let $\left\{x_{n}\right\}$ be a sequence in $X$. If $d\left(x_{n+1}, p\right) \leq d\left(x_{n}, p\right)$ for all $p \in C$ and $n \in \mathbb{N}$, then $\left\{\pi x_{n}\right\}$ converges strongly to some element in $C$.

Proof Let $m>n$. By the $(\mathrm{CN})$ inequality and the property of $\pi$, it follows that

$$
\begin{aligned}
d\left(\pi x_{m}, \pi x_{n}\right)^{2} & \leq 2 d\left(x_{m}, \pi x_{m}\right)^{2}+2 d\left(x_{m}, \pi x_{n}\right)^{2}-4 d\left(x_{m}, \frac{\pi x_{m} \oplus \pi x_{n}}{2}\right)^{2} \\
& \leq 2 d\left(x_{m}, \pi x_{m}\right)^{2}+2 d\left(x_{m}, \pi x_{n}\right)^{2}-4 d\left(x_{m}, \pi x_{m}\right)^{2} \\
& =2 d\left(x_{m}, \pi x_{n}\right)^{2}-2 d\left(x_{m}, \pi x_{m}\right)^{2} \\
& \leq 2 d\left(x_{n}, \pi x_{n}\right)^{2}-2 d\left(x_{m}, \pi x_{m}\right)^{2}
\end{aligned}
$$

This implies that

$$
d\left(x_{m}, \pi x_{m}\right)^{2} \leq d\left(x_{n}, \pi x_{n}\right)^{2} \quad \text { for } m>n
$$

Then $\lim _{n \rightarrow \infty} d\left(x_{n}, \pi x_{n}\right)^{2}$ exists. Letting $m, n \rightarrow \infty$ in (1.2), we have that $\left\{\pi x_{n}\right\}$ is a Cauchy sequence in a closed subset $C$ of a complete CAT(0) space $X$, hence it converges to some element in $C$.

Let $C$ be a nonempty closed and convex subset of a Hilbert space $H$. A mapping $T: C \rightarrow$ $C$ is called generalized hybrid if there exist $\alpha, \beta \in \mathbb{R}$ such that

$$
\alpha\|T x-T y\|^{2}+(1-\alpha)\|x-T y\|^{2} \leq \beta\|T x-y\|^{2}+(1-\beta)\|x-y\|^{2}
$$

for all $x, y \in C$. We note that the generalized hybrid mappings generalize several wellknown mappings. For example, a generalized hybrid mapping is nonexpansive for $\alpha=1$ 
and $\beta=0$, nonspreading for $\alpha=2$ and $\beta=1$, and hybrid for $\alpha=\frac{3}{2}$ and $\beta=\frac{1}{2}$. In 2010, Kocourek et al. [32] proved the fixed point theorems for generalized hybrid mappings in Hilbert spaces. Later in 2011, Takahashi and Yao [33] extended the results of Kocourek et al. to uniformly convex Banach spaces.

Recently, Maruyama et al. [34] introduced a new nonlinear mapping in a Hilbert space as follows. Let $N \in \mathbb{N}$. A mapping $T: C \rightarrow C$ is called $N$-generalized hybrid if there are $\alpha_{1}, \ldots, \alpha_{N}, \beta_{1}, \ldots, \beta_{N} \in \mathbb{R}$ such that

$$
\begin{aligned}
& \sum_{k=1}^{N} \alpha_{k}\left\|T^{N+1-k} x-T y\right\|^{2}+\left(1-\sum_{k=1}^{N} \alpha_{k}\right)\|x-T y\|^{2} \\
& \leq \sum_{k=1}^{N} \beta_{k}\left\|T^{N+1-k} x-y\right\|^{2}+\left(1-\sum_{k=1}^{N} \beta_{k}\right)\|x-y\|^{2}
\end{aligned}
$$

for all $x, y \in C$. They obtained the existence and weak convergence theorems for $N$-generalized hybrid mappings in Hilbert spaces. Hojo et al. [35] also studied the fixed point theorems for $N$-generalized hybrid mappings in Hilbert spaces and provided an example of $\mathrm{N}$-generalized hybrid mappings which are not generalized hybrid mappings as follows.

Example 1.7 Let $H$ be a Hilbert space, $A=\{x \in H:\|x\| \leq 1\}$ and define a mapping $T$ : $H \rightarrow H$ as follows:

$$
T x= \begin{cases}0 & \text { for all } x \in A ; \\ \frac{x}{\|x\|} & \text { for all } x \notin A .\end{cases}
$$

We observe that the $\mathrm{N}$-generalized hybrid mappings generalize several well-known mappings, for instance, nonexpansive mappings, nonspreading mappings, hybrid mappings, $\lambda$-hybrid mappings, generalized hybrid mappings, and 2-generalized hybrid mappings. Many researchers have studied the fixed point theorems of those mappings in both Hilbert spaces and Banach spaces (e.g., see [32, 33, 36-38]). However, no researcher has studied the fixed point theorems for $\mathrm{N}$-generalized hybrid mappings in more general spaces. So, in this paper, we are interested in studying and extending those mappings to both uniformly convex metric spaces and $\mathrm{CAT}(0)$ spaces.

\section{Fixed point theorems in uniformly convex metric spaces}

We first define $N$-generalized hybrid mappings in convex metric spaces. Let $C$ be a nonempty subset of a convex metric space $(X, d, W)$. Let $N \in \mathbb{N}$. A mapping $T: C \rightarrow C$ is called $N$-generalized hybrid if there are $\alpha_{1}, \ldots, \alpha_{N}, \beta_{1}, \ldots, \beta_{N} \in \mathbb{R}$ such that

$$
\begin{gathered}
\sum_{k=1}^{N} \alpha_{k} d\left(T^{N+1-k} x, T y\right)^{2}+\left(1-\sum_{k=1}^{N} \alpha_{k}\right) d(x, T y)^{2} \\
\leq \sum_{k=1}^{N} \beta_{k} d\left(T^{N+1-k} x, y\right)^{2}+\left(1-\sum_{k=1}^{N} \beta_{k}\right) d(x, y)^{2}
\end{gathered}
$$

for all $x, y \in C$. Now, we prove a fixed point theorem for $N$-generalized hybrid mappings in complete uniformly convex metric spaces. 
Theorem 2.1 Let $C$ be a nonempty closed and convex subset of a complete uniformly convex metric space $(X, d, W)$ and let $T: C \rightarrow C$ be an $N$-generalized hybrid mapping with $\sum_{k=1}^{N} \alpha_{k} \in(-\infty, 0] \cup[1, \infty)$ and $\sum_{k=1}^{N} \beta_{k} \in[0,1]$. Then $T$ has a fixed point if and only if there exists an $x \in C$ such that $\left\{T^{n} x\right\}$ is bounded.

Proof The necessity is obvious. Conversely, we assume that there exists an $x \in C$ such that $\left\{T^{n} x\right\}$ is bounded. We will show that $F(T)$ is nonempty. From Lemma $1.2, A\left(C,\left\{T^{n} x\right\}\right)$ is a singleton set. Let $A\left(C,\left\{T^{n} x\right\}\right)=\{z\}$. Since $T$ is $N$-generalized hybrid, there are $\alpha_{1}, \ldots, \alpha_{N}, \beta_{1}, \ldots, \beta_{N} \in \mathbb{R}$ such that

$$
\begin{gathered}
\sum_{k=1}^{N} \alpha_{k} d\left(T^{n+N+1-k} x, T z\right)^{2}+\left(1-\sum_{k=1}^{N} \alpha_{k}\right) d\left(T^{n} x, T z\right)^{2} \\
\leq \sum_{k=1}^{N} \beta_{k} d\left(T^{n+N+1-k} x, z\right)^{2}+\left(1-\sum_{k=1}^{N} \beta_{k}\right) d\left(T^{n} x, z\right)^{2} .
\end{gathered}
$$

If $\sum_{k=1}^{N} \alpha_{k} \in[1, \infty)$ and $\sum_{k=1}^{N} \beta_{k} \in[0,1]$, then (2.1) becomes

$$
\begin{aligned}
\sum_{k=1}^{N} \alpha_{k} d\left(T^{n+N+1-k} x, T z\right)^{2} \leq & \sum_{k=1}^{N} \beta_{k} d\left(T^{n+N+1-k} x, z\right)^{2}+\left(1-\sum_{k=1}^{N} \beta_{k}\right) d\left(T^{n} x, z\right)^{2} \\
& +\left(\sum_{k=1}^{N} \alpha_{k}-1\right) d\left(T^{n} x, T z\right)^{2} .
\end{aligned}
$$

This implies that

$$
\limsup _{n \rightarrow \infty} d\left(T^{n} x, T z\right)^{2} \leq \limsup _{n \rightarrow \infty} d\left(T^{n} x, z\right)^{2}
$$

If $\sum_{k=1}^{N} \alpha_{k} \in(-\infty, 0]$ and $\sum_{k=1}^{N} \beta_{k} \in[0,1]$, then (2.1) becomes

$$
\begin{aligned}
\left(1-\sum_{k=1}^{N} \alpha_{k}\right) d\left(T^{n} x, T z\right)^{2} \leq & \sum_{k=1}^{N} \beta_{k} d\left(T^{n+N+1-k} x, z\right)^{2}+\left(1-\sum_{k=1}^{N} \beta_{k}\right) d\left(T^{n} x, z\right)^{2} \\
& -\sum_{k=1}^{N} \alpha_{k} d\left(T^{n+N+1-k} x, T z\right)^{2}
\end{aligned}
$$

This implies again that

$$
\limsup _{n \rightarrow \infty} d\left(T^{n} x, T z\right)^{2} \leq \limsup _{n \rightarrow \infty} d\left(T^{n} x, z\right)^{2}
$$

Therefore, we have

$$
r\left(T z,\left\{T^{n} x\right\}\right) \leq r\left(z,\left\{T^{n} x\right\}\right)
$$

Since $T z \in C$ and $r\left(z,\left\{T^{n} x\right\}\right)=\inf \left\{r\left(y,\left\{T^{n} x\right\}\right): y \in C\right\}$, it implies that $T z=z$. Hence, $F(T)$ is nonempty. 
As a direct consequence of Theorem 2.1, we obtain a fixed point theorem for $N$-generalized hybrid mappings in uniformly convex metric spaces as follows.

Theorem 2.2 Let $C$ be a nonempty bounded closed and convex subset of a complete uniformly convex metric space $(X, d, W)$ and let $T: C \rightarrow C$ be an $N$-generalized hybrid mapping with $\sum_{k=1}^{N} \alpha_{k} \in(-\infty, 0] \cup[1, \infty)$ and $\sum_{k=1}^{N} \beta_{k} \in[0,1]$. Then $T$ has a fixed point.

We can show that if $T$ is an $N$-generalized hybrid mapping and $x=T x$, then for any $y \in C$, we get

$$
\sum_{k=1}^{N} \alpha_{k} d(x, T y)^{2}+\left(1-\sum_{k=1}^{N} \alpha_{k}\right) d(x, T y)^{2} \leq \sum_{k=1}^{N} \beta_{k} d(x, y)^{2}+\left(1-\sum_{k=1}^{N} \beta_{k}\right) d(x, y)^{2}
$$

and hence $d(x, T y) \leq d(x, y)$. This means that an $N$-generalized hybrid mapping with a fixed point is quasi-nonexpansive. Then, using the methods of the proof of Theorem 1.3 in [13], we can prove the following.

Corollary 2.3 Let $C$ be a nonempty convex subset of a complete uniformly convex metric space $(X, d, W)$. Suppose that $T: C \rightarrow C$ is an $N$-generalized hybrid mapping and has a fixed point. Then $F(T)$ is closed and convex.

\section{Remark 2.4}

(i) Theorems 2.1 and 2.2 extend and generalize the corresponding results in [17, 32-34, 36-38] to $N$-generalized hybrid mappings on uniformly convex metric spaces.

(ii) In CAT(0) spaces, if we set $W(x, y, \lambda):=\lambda x \oplus(1-\lambda) y$, then Theorems 2.1 and 2.2 can be applied to these spaces under the assumption that $\sum_{k=1}^{N} \alpha_{k} \in(-\infty, 0] \cup[1, \infty)$ and $\sum_{k=1}^{N} \beta_{k} \in[0,1]$.

\section{Fixed point theorems in CAT(0) spaces}

In this section, we study the existence and $\Delta$-convergence theorems for $N$-generalized hybrid mappings in complete CAT $(0)$ spaces.

We first recall the definition of a Banach limit. Let $\mu$ be a continuous linear functional on $l^{\infty}$, the Banach space of bounded real sequences, and $\left(a_{1}, a_{2}, \ldots\right) \in l^{\infty}$. We write $\mu_{n}\left(a_{n}\right)$ instead of $\mu\left(\left(a_{1}, a_{2}, \ldots\right)\right)$. We call $\mu$ a Banach limit if $\mu$ satisfies $\|\mu\|=\mu(1,1, \ldots)=1$ and $\mu_{n}\left(a_{n}\right)=\mu_{n}\left(a_{n+1}\right)$ for each $\left(a_{1}, a_{2}, \ldots\right) \in l^{\infty}$. For a Banach limit $\mu$, we know that $\liminf _{n \rightarrow \infty} a_{n} \leq \mu_{n}\left(a_{n}\right) \leq \limsup _{n \rightarrow \infty} a_{n}$ for all $\left(a_{1}, a_{2}, \ldots\right) \in l^{\infty}$. So if $\left(a_{1}, a_{2}, \ldots\right) \in l^{\infty}$ with $\lim _{n \rightarrow \infty} a_{n}=c$, then $\mu_{n}\left(a_{n}\right)=c$; see [39] for more details.

Now, we obtain the following lemma in $\mathrm{CAT}(0)$ spaces.

Lemma 3.1 Let $C$ be a nonempty closed and convex subset of a complete CAT(0) space $X$, let $\left\{x_{n}\right\}$ be a bounded sequence in $X$, and let $\mu$ be a Banach limit. If a function $f: C \rightarrow \mathbb{R}$ is defined by

$$
f(z)=\mu_{n} d\left(x_{n}, z\right)^{2} \quad \text { for all } z \in C,
$$

then there exists a unique $z_{0} \in C$ such that

$$
f\left(z_{0}\right)=\min \{f(z): z \in C\} .
$$


Proof It is easy to show that $f$ is continuous. By $(\mathrm{CN})$ inequality, we obtain that

$$
f\left(\frac{x \oplus y}{2}\right) \leq \frac{1}{2} f(x)+\frac{1}{2} f(y)-\frac{1}{4} d(x, y)^{2} \quad \text { for all } x, y \in C .
$$

This implies by Proposition 1.7 in [40] that there exists a unique $z_{0} \in C$ such that $f\left(z_{0}\right)=$ $\min \{f(z): z \in C\}$.

By using Lemma 3.1, we can prove the following fixed point theorem for $N$-generalized hybrid mappings in $\mathrm{CAT}(0)$ spaces without the assumptions that $\sum_{k=1}^{N} \alpha_{k} \in(-\infty, 0] \cup$ $[1, \infty)$ and $\sum_{k=1}^{N} \beta_{k} \in[0,1]$.

Theorem 3.2 Let $C$ be a nonempty closed and convex subset of a complete CAT(0) space $X$ and let $T: C \rightarrow C$ be an $N$-generalized hybrid mapping. Then $T$ has a fixed point if and only if there exists an $x \in C$ such that $\left\{T^{n} x\right\}$ is bounded.

Proof The necessity is obvious. Conversely, we assume that there exists an $x \in C$ such that $\left\{T^{n} x\right\}$ is bounded. Let $\mu$ be a Banach limit. Since $T$ is $N$-generalized hybrid, there are $\alpha_{1}, \ldots, \alpha_{N}, \beta_{1}, \ldots, \beta_{N} \in \mathbb{R}$ such that

$$
\begin{aligned}
& \sum_{k=1}^{N} \alpha_{k} d\left(T^{n+N+1-k} x, T z\right)^{2}+\left(1-\sum_{k=1}^{N} \alpha_{k}\right) d\left(T^{n} x, T z\right)^{2} \\
& \leq \sum_{k=1}^{N} \beta_{k} d\left(T^{n+N+1-k} x, z\right)^{2}+\left(1-\sum_{k=1}^{N} \beta_{k}\right) d\left(T^{n} x, z\right)^{2}
\end{aligned}
$$

for any $z \in C$ and $n \in \mathbb{N} \cup\{0\}$. Since $\left\{T^{n} x\right\}$ is bounded, we have

$$
\begin{aligned}
& \sum_{k=1}^{N} \alpha_{k} \mu_{n} d\left(T^{n+N+1-k} x, T z\right)^{2}+\left(1-\sum_{k=1}^{N} \alpha_{k}\right) \mu_{n} d\left(T^{n} x, T z\right)^{2} \\
& \leq \sum_{k=1}^{N} \beta_{k} \mu_{n} d\left(T^{n+N+1-k} x, z\right)^{2}+\left(1-\sum_{k=1}^{N} \beta_{k}\right) \mu_{n} d\left(T^{n} x, z\right)^{2} .
\end{aligned}
$$

This implies that

$$
\mu_{n} d\left(T^{n} x, T z\right)^{2} \leq \mu_{n} d\left(T^{n} x, z\right)^{2}
$$

for all $z \in C$. It follows by Lemma 3.1 that $T z=z$. Hence, $F(T)$ is nonempty.

As a direct consequence of Theorem 3.2, we obtain a fixed point theorem for $N$-generalized hybrid mappings in $\mathrm{CAT}(0)$ spaces as follows.

Theorem 3.3 Let $C$ be a nonempty bounded closed and convex subset of a complete CAT(0) space $X$ and let $T: C \rightarrow C$ be an $N$-generalized hybrid mapping. Then $T$ has a fixed point.

Remark 3.4 Theorems 3.2 and 3.3 extend and generalize the corresponding results in [17, $32-34,36-38$ ] to $N$-generalized hybrid mappings on CAT(0) spaces.

Next, we study the $\Delta$-convergence theorem for $N$-generalized hybrid mappings in $\mathrm{CAT}(0)$ spaces. Before proving the theorem, we need the following lemma. 
Lemma 3.5 Let $C$ be a nonempty closed and convex subset of a complete $\mathrm{CAT}(0)$ space $X$ and let $T: C \rightarrow C$ be an $N$-generalized hybrid mapping with $\sum_{k=1}^{N} \alpha_{k} \in(-\infty, 0] \cup[1, \infty)$ and $\sum_{k=1}^{N} \beta_{k} \in[0, \infty)$. If $\left\{x_{n}\right\}$ is a bounded sequence in $C$ with $\Delta-\lim _{n \rightarrow \infty} x_{n}=x$ and $\lim _{n \rightarrow \infty} d\left(x_{n}, T^{i} x_{n}\right)=0$ for all $i=1,2, \ldots, N$, then $x \in F(T)$.

Proof Since $T$ is an $N$-generalized hybrid mapping, there are $\alpha_{1}, \ldots, \alpha_{N}, \beta_{1}, \ldots, \beta_{N} \in \mathbb{R}$ such that

$$
\begin{aligned}
& \sum_{k=1}^{N} \alpha_{k} d\left(T^{N+1-k} x_{n}, T x\right)^{2}+\left(1-\sum_{k=1}^{N} \alpha_{k}\right) d\left(x_{n}, T x\right)^{2} \\
& \leq \sum_{k=1}^{N} \beta_{k} d\left(T^{N+1-k} x_{n}, x\right)^{2}+\left(1-\sum_{k=1}^{N} \beta_{k}\right) d\left(x_{n}, x\right)^{2} .
\end{aligned}
$$

Case 1: $\sum_{k=1}^{N} \alpha_{k} \in[1, \infty)$ and $\sum_{k=1}^{N} \beta_{k} \in[0, \infty)$. It follows by (3.1) that

$$
\begin{aligned}
\sum_{k=1}^{N} \alpha_{k} d\left(T^{N+1-k} x_{n}, T x\right)^{2} \\
\leq \sum_{k=1}^{N} \beta_{k} d\left(T^{N+1-k} x_{n}, x\right)^{2}+\left(1-\sum_{k=1}^{N} \beta_{k}\right) d\left(x_{n}, x\right)^{2}+\left(\sum_{k=1}^{N} \alpha_{k}-1\right) d\left(x_{n}, T x\right)^{2} \\
\leq \sum_{k=1}^{N} \beta_{k}\left(d\left(T^{N+1-k} x_{n}, x_{n}\right)^{2}+2 d\left(T^{N+1-k} x_{n}, x_{n}\right) d\left(x_{n}, x\right)+d\left(x_{n}, x\right)^{2}\right) \\
\quad+\left(1-\sum_{k=1}^{N} \beta_{k}\right) d\left(x_{n}, x\right)^{2}+\left(\sum_{k=1}^{N} \alpha_{k}-1\right)\left(d\left(x_{n}, T^{N+1-k} x_{n}\right)^{2}\right. \\
\left.\quad+2 d\left(x_{n}, T^{N+1-k} x_{n}\right) d\left(T^{N+1-k} x_{n}, T x\right)+d\left(T^{N+1-k} x_{n}, T x\right)^{2}\right) \\
=d\left(x_{n}, x\right)^{2}+\left(\sum_{k=1}^{N} \beta_{k}+\sum_{k=1}^{N} \alpha_{k}-1\right) d\left(T^{N+1-k} x_{n}, x_{n}\right)^{2} \\
\quad+2 \sum_{k=1}^{N} \beta_{k} d\left(T^{N+1-k} x_{n}, x_{n}\right) d\left(x_{n}, x\right) \\
\quad+2\left(\sum_{k=1}^{N} \alpha_{k}-1\right) d\left(x_{n}, T^{N+1-k} x_{n}\right) d\left(T^{N+1-k} x_{n}, T x\right) \\
+\left(\sum_{k=1}^{N} \alpha_{k}-1\right) d\left(T^{N+1-k} x_{n}, T x\right)^{2} .
\end{aligned}
$$

This implies that

$$
\begin{aligned}
& d\left(T^{N+1-k} x_{n}, T x\right)^{2} \\
& \quad \leq d\left(x_{n}, x\right)^{2}+\left(\sum_{k=1}^{N} \beta_{k}+\sum_{k=1}^{N} \alpha_{k}-1\right) d\left(T^{N+1-k} x_{n}, x_{n}\right)^{2}
\end{aligned}
$$




$$
\begin{aligned}
& +2 \sum_{k=1}^{N} \beta_{k} d\left(T^{N+1-k} x_{n}, x_{n}\right) d\left(x_{n}, x\right) \\
& +2\left(\sum_{k=1}^{N} \alpha_{k}-1\right) d\left(x_{n}, T^{N+1-k} x_{n}\right) d\left(T^{N+1-k} x_{n}, T x\right) .
\end{aligned}
$$

Since $\left\{x_{n}\right\}$ is bounded and $\lim _{n \rightarrow \infty} d\left(x_{n}, T^{i} x_{n}\right)=0$ for all $i=1,2, \ldots, N$, we have that $\left\{T x_{n}\right\},\left\{T^{2} x_{n}\right\}, \ldots,\left\{T^{N} x_{n}\right\}$ are bounded. So, we have

$$
\begin{aligned}
& d\left(T^{N+1-k} x_{n}, T x\right)^{2} \\
& \leq d\left(x_{n}, x\right)^{2}+\left(\sum_{k=1}^{N} \beta_{k}+\sum_{k=1}^{N} \alpha_{k}-1\right) d\left(T^{N+1-k} x_{n}, x_{n}\right)^{2} \\
& \quad+2 \sum_{k=1}^{N} \beta_{k} d\left(T^{N+1-k} x_{n}, x_{n}\right) M+2\left(\sum_{k=1}^{N} \alpha_{k}-1\right) d\left(x_{n}, T^{N+1-k} x_{n}\right) M \\
& =d\left(x_{n}, x\right)^{2}+\left(\sum_{k=1}^{N} \beta_{k}+\sum_{k=1}^{N} \alpha_{k}-1\right) d\left(T^{N+1-k} x_{n}, x_{n}\right)\left(d\left(T^{N+1-k} x_{n}, x_{n}\right)+2 M\right)
\end{aligned}
$$

where $M=\max _{1 \leq k \leq N} \sup \left\{d\left(x_{n}, x\right), d\left(T^{N+1-k} x_{n}, T x\right): n \in \mathbb{N}\right\}$.

Case 2: $\sum_{k=1}^{N} \alpha_{k} \in(-\infty, 0]$ and $\sum_{k=1}^{N} \beta_{k} \in[0, \infty)$. In the same way as Case 1 , we can show that

$$
\begin{aligned}
& d\left(T^{N+1-k} x_{n}, T x\right)^{2} \\
& \quad \leq d\left(x_{n}, x\right)^{2}+\left(\sum_{k=1}^{N} \beta_{k}-\sum_{k=1}^{N} \alpha_{k}\right) d\left(T^{N+1-k} x_{n}, x_{n}\right)\left(d\left(T^{N+1-k} x_{n}, x_{n}\right)+2 M\right) .
\end{aligned}
$$

By Case 1, Case 2, and the assumption $\lim _{n \rightarrow \infty} d\left(x_{n}, T^{i} x_{n}\right)=0$ for all $i=1,2, \ldots, N$, we obtain

$$
\limsup _{n \rightarrow \infty} d\left(x_{n}, T x\right) \leq \limsup _{n \rightarrow \infty} d\left(x_{n}, x\right)
$$

Since $\Delta-\lim _{n \rightarrow \infty} x_{n}=x$, it follows by the uniqueness of asymptotic centers that $T x=x$. Hence, $x \in F(T)$.

Fixed point iteration methods are very useful for approximating a fixed point of various nonlinear mappings such as Mann iteration, Ishikawa iteration, Noor iteration and so on. We now introduce a new iteration method for approximating a fixed point of mappings in a CAT(0) space $X$ as follows: Let $C$ be a nonempty closed and convex subset of $X$, let $T: C \rightarrow C$ be a mapping and $N \in \mathbb{N}$. For $x_{1} \in C$, the sequence $\left\{x_{n}\right\}$ generated by

$$
x_{n+1}=\bigoplus_{i=0}^{N} \lambda_{n}^{(i)} T^{i} x_{n} \quad \text { for all } n \in \mathbb{N}
$$

where $\left\{\lambda_{n}^{(i)}\right\}$ is a sequence in $[0,1]$ for all $i=0,1, \ldots, N$ with $\sum_{i=0}^{N} \lambda_{n}^{(i)}=1$. 
Remark 3.6 If we put

$$
W_{n}^{(N)}=\bigoplus_{i=0}^{N} \frac{\lambda_{n}^{(i)}}{\sum_{j=0}^{N} \lambda_{n}^{(j)}} T^{i} x_{n}
$$

then by (1.1) we get

$$
W_{n}^{(N)}=\frac{\sum_{j=0}^{N-1} \lambda_{n}^{(j)}}{\sum_{j=0}^{N} \lambda_{n}^{(j)}} W_{n}^{(N-1)} \oplus \frac{\lambda_{n}^{(N)}}{\sum_{j=0}^{N} \lambda_{n}^{(j)}} T^{N} x_{n} .
$$

Indeed, we put $\delta_{n}^{(i, N)}=\frac{\lambda_{n}^{(i)}}{\sum_{j=0}^{N} \lambda_{n}^{(j)}}$ for $i=0,1, \ldots, N$. Thus

$$
\begin{aligned}
W_{n}^{(N)}= & \bigoplus_{i=0}^{N} \frac{\lambda_{n}^{(i)}}{\sum_{j=0}^{N} \lambda_{n}^{(j)}} T^{i} x_{n}=\bigoplus_{i=0}^{N} \delta_{n}^{(i, N)} T^{i} x_{n} \\
= & \left(1-\delta_{n}^{(N, N)}\right)\left(\frac{\delta_{n}^{(0, N)}}{1-\delta_{n}^{(N, N)}} x_{n} \oplus \frac{\delta_{n}^{(1, N)}}{1-\delta_{n}^{(N, N)}} T x_{n} \oplus \cdots \oplus \frac{\delta_{n}^{(N-1, N)}}{1-\delta_{n}^{(N, N)}} T^{N-1} x_{n}\right) \\
& \oplus \delta_{n}^{(N, N)} T^{N} x_{n} \\
= & \left(1-\delta_{n}^{(N, N)}\right)\left(\delta_{n}^{(0, N-1)} x_{n} \oplus \delta_{n}^{(1, N-1)} T x_{n} \oplus \cdots \oplus \delta_{n}^{(N-1, N-1)} T^{N-1} x_{n}\right) \oplus \delta_{n}^{(N, N)} T^{N} x_{n} \\
= & \left(1-\delta_{n}^{(N, N)}\right)\left(\frac{\lambda_{n}^{(0)}}{\sum_{j=0}^{N-1} \lambda_{n}^{(j)}} x_{n} \oplus \frac{\lambda_{n}^{(1)}}{\sum_{j=0}^{N-1} \lambda_{n}^{(j)}} T x_{n} \oplus \cdots \oplus \frac{\lambda_{n}^{(N-1)}}{\sum_{j=0}^{N-1} \lambda_{n}^{(j)}} T^{N-1} x_{n}\right) \\
& \oplus \delta_{n}^{(N, N)} T^{N} x_{n} \\
= & \left(1-\delta_{n}^{(N, N)}\right) W_{n}^{(N-1)} \oplus \delta_{n}^{(N, N)} T^{N} x_{n} \\
= & \frac{\sum_{j=0}^{N-1} \lambda_{n}^{(j)}}{\sum_{j=0}^{N} \lambda_{n}^{(j)}} W_{n}^{(N-1)} \oplus \frac{\lambda_{n}^{(N)}}{\sum_{j=0}^{N} \lambda_{n}^{(j)}} T^{N} x_{n} .
\end{aligned}
$$

Therefore, (3.3) is justified.

Using Lemma 3.5 , we can prove the $\Delta$-convergence theorem for $N$-generalized hybrid mappings in complete $\mathrm{CAT}(0)$ spaces as follows.

Theorem 3.7 Let $C$ be a nonempty closed and convex subset of a complete $\mathrm{CAT}(0)$ space $X$ and let $T: C \rightarrow C$ be an $N$-generalized hybrid mapping with $F(T) \neq \emptyset$ and $\sum_{k=1}^{N} \alpha_{k} \in$ $(-\infty, 0] \cup[1, \infty)$ and $\sum_{k=1}^{N} \beta_{k} \in[0, \infty)$. Let $\pi: C \rightarrow F(T)$ be the nearest point projection mapping. Suppose that $\left\{x_{n}\right\}$ is a sequence in $C$ defined by (3.2) with $0<a \leq \lambda_{n}^{(i)} \leq b<1$ for all $i=0,1, \ldots, N$. Then $\left\{x_{n}\right\} \Delta$-converges to a fixed point $u$ of $T$, where $u=\lim _{n \rightarrow \infty} \pi x_{n}$.

Proof Since $T$ is $N$-generalized hybrid and $F(T) \neq \emptyset$, we get $T$ is quasi-nonexpansive. Then, for $p \in F(T)$, we have

$$
\begin{aligned}
d\left(x_{n+1}, p\right) & =d\left(\bigoplus_{i=0}^{N} \lambda_{n}^{(i)} T^{i} x_{n}, p\right) \\
& \leq \sum_{i=0}^{N} \lambda_{n}^{(i)} d\left(T^{i} x_{n}, p\right)
\end{aligned}
$$




$$
\begin{aligned}
& \leq \sum_{i=0}^{N} \lambda_{n}^{(i)} d\left(x_{n}, p\right) \\
& =d\left(x_{n}, p\right) .
\end{aligned}
$$

Therefore, $\lim _{n \rightarrow \infty} d\left(x_{n}, p\right)$ exists and hence $\left\{x_{n}\right\}$ is bounded.

For each $p \in F(T)$, we obtain, by (3.2), (3.3), and the ( $\left.\mathrm{CN}^{*}\right)$ inequality, that

$$
\begin{aligned}
& d\left(x_{n+1}, p\right)^{2} \\
& =d\left(\bigoplus_{i=0}^{N} \frac{\lambda_{n}^{(i)}}{\sum_{j=0}^{N} \lambda_{n}^{(j)}} T^{i} x_{n}, p\right)^{2}=d\left(W_{n}^{(N)}, p\right)^{2} \\
& =d\left(\frac{\sum_{j=0}^{N-1} \lambda_{n}^{(j)}}{\sum_{j=0}^{N} \lambda_{n}^{(j)}} W_{n}^{(N-1)} \oplus \frac{\lambda_{n}^{(N)}}{\sum_{j=0}^{N} \lambda_{n}^{(j)}} T^{N} x_{n}, p\right)^{2} \\
& \leq \frac{\sum_{j=0}^{N-1} \lambda_{n}^{(j)}}{\sum_{j=0}^{N} \lambda_{n}^{(j)}} d\left(W_{n}^{(N-1)}, p\right)^{2}+\frac{\lambda_{n}^{(N)}}{\sum_{j=0}^{N} \lambda_{n}^{(j)}} d\left(T^{N} x_{n}, p\right)^{2} \\
& -\frac{\lambda_{n}^{(N)}}{\sum_{j=0}^{N} \lambda_{n}^{(j)}} \frac{\sum_{j=0}^{N-1} \lambda_{n}^{(j)}}{\sum_{j=0}^{N} \lambda_{n}^{(j)}} d\left(W_{n}^{(N-1)}, T^{N} x_{n}\right)^{2} \\
& =\sum_{j=0}^{N-1} \lambda_{n}^{(j)} d\left(W_{n}^{(N-1)}, p\right)^{2}+\lambda_{n}^{(N)} d\left(T^{N} x_{n}, p\right)^{2}-\lambda_{n}^{(N)} \sum_{j=0}^{N-1} \lambda_{n}^{(j)} d\left(W_{n}^{(N-1)}, T^{N} x_{n}\right)^{2} \\
& =\sum_{j=0}^{N-1} \lambda_{n}^{(j)} d\left(\frac{\sum_{j=0}^{N-2} \lambda_{n}^{(j)}}{\sum_{j=0}^{N-1} \lambda_{n}^{(j)}} W_{n}^{(N-2)} \oplus \frac{\lambda_{n}^{(N-1)}}{\sum_{j=0}^{N-1} \lambda_{n}^{(j)}} T^{N-1} x_{n}, p\right)^{2}+\lambda_{n}^{(N)} d\left(T^{N} x_{n}, p\right)^{2} \\
& -\lambda_{n}^{(N)} \sum_{j=0}^{N-1} \lambda_{n}^{(j)} d\left(W_{n}^{(N-1)}, T^{N} x_{n}\right)^{2} \\
& \leq \sum_{j=0}^{N-1} \lambda_{n}^{(j)}\left(\frac{\sum_{j=0}^{N-2} \lambda_{n}^{(j)}}{\sum_{j=0}^{N-1} \lambda_{n}^{(j)}} d\left(W_{n}^{(N-2)}, p\right)^{2}+\frac{\lambda_{n}^{(N-1)}}{\sum_{j=0}^{N-1} \lambda_{n}^{(j)}} d\left(T^{N-1} x_{n}, p\right)^{2}\right. \\
& \left.-\frac{\sum_{j=0}^{N-2} \lambda_{n}^{(j)}}{\sum_{j=0}^{N-1} \lambda_{n}^{(j)}} \frac{\lambda_{n}^{(N-1)}}{\sum_{j=0}^{N-1} \lambda_{n}^{(j)}} d\left(W_{n}^{(N-2)}, T^{N-1} x_{n}\right)^{2}\right)+\lambda_{n}^{(N)} d\left(T^{N} x_{n}, p\right)^{2} \\
& -\lambda_{n}^{(N)} \sum_{j=0}^{N-1} \lambda_{n}^{(j)} d\left(W_{n}^{(N-1)}, T^{N} x_{n}\right)^{2} \\
& =\sum_{j=0}^{N-2} \lambda_{n}^{(j)} d\left(W_{n}^{(N-2)}, p\right)^{2}+\lambda_{n}^{(N-1)} d\left(T^{N-1} x_{n}, p\right)^{2}+\lambda_{n}^{(N)} d\left(T^{N} x_{n}, p\right)^{2} \\
& -\frac{\lambda_{n}^{(N-1)} \sum_{j=0}^{N-2} \lambda_{n}^{(j)}}{\sum_{j=0}^{N-1} \lambda_{n}^{(j)}} d\left(W_{n}^{(N-2)}, T^{N-1} x_{n}\right)^{2}-\lambda_{n}^{(N)} \sum_{j=0}^{N-1} \lambda_{n}^{(j)} d\left(W_{n}^{(N-1)}, T^{N} x_{n}\right)^{2} \\
& \leq \sum_{j=0}^{N-3} \lambda_{n}^{(j)} d\left(W_{n}^{(N-3)}, p\right)^{2}+\lambda_{n}^{(N-2)} d\left(T^{N-2} x_{n}, p\right)^{2}+\lambda_{n}^{(N-1)} d\left(T^{N-1} x_{n}, p\right)^{2} \\
& +\lambda_{n}^{(N)} d\left(T^{N} x_{n}, p\right)^{2}-\frac{\lambda_{n}^{(N-2)} \sum_{j=0}^{N-3} \lambda_{n}^{(j)}}{\sum_{j=0}^{N-2} \lambda_{n}^{(j)}} d\left(W_{n}^{(N-3)}, T^{N-2} x_{n}\right)^{2}
\end{aligned}
$$




$$
\begin{aligned}
& -\frac{\lambda_{n}^{(N-1)} \sum_{j=0}^{N-2} \lambda_{n}^{(j)}}{\sum_{j=0}^{N-1} \lambda_{n}^{(j)}} d\left(W_{n}^{(N-2)}, T^{N-1} x_{n}\right)^{2}-\lambda_{n}^{(N)} \sum_{j=0}^{N-1} \lambda_{n}^{(j)} d\left(W_{n}^{(N-1)}, T^{N} x_{n}\right)^{2} \\
\vdots & \\
\leq & \lambda_{n}^{(0)} d\left(W_{n}^{(0)}, p\right)^{2}+\sum_{k=1}^{N} \lambda_{n}^{(k)} d\left(T^{k} x_{n}, p\right)^{2}-\sum_{k=1}^{N} \frac{\lambda_{n}^{(k)} \sum_{j=0}^{k-1} \lambda_{n}^{(j)}}{\sum_{j=0}^{k} \lambda_{n}^{(j)}} d\left(W_{n}^{(k-1)}, T^{k} x_{n}\right)^{2} \\
\leq & \sum_{k=0}^{N} \lambda_{n}^{(k)} d\left(x_{n}, p\right)^{2}-\sum_{k=1}^{N} \frac{\lambda_{n}^{(k)} \sum_{j=0}^{k-1} \lambda_{n}^{(j)}}{\sum_{j=0}^{k} \lambda_{n}^{(j)}} d\left(W_{n}^{(k-1)}, T^{k} x_{n}\right)^{2} \\
= & d\left(x_{n}, p\right)^{2}-\sum_{k=1}^{N} \frac{\lambda_{n}^{(k)} \sum_{j=0}^{k-1} \lambda_{n}^{(j)}}{\sum_{j=0}^{k} \lambda_{n}^{(j)}} d\left(W_{n}^{(k-1)}, T^{k} x_{n}\right)^{2} .
\end{aligned}
$$

This implies that

$$
\sum_{k=1}^{N} \frac{\lambda_{n}^{(k)} \sum_{j=0}^{k-1} \lambda_{n}^{(j)}}{\sum_{j=0}^{k} \lambda_{n}^{(j)}} d\left(W_{n}^{(k-1)}, T^{k} x_{n}\right)^{2} \leq d\left(x_{n}, p\right)^{2}-d\left(x_{n+1}, p\right)^{2} .
$$

Since $\lim _{n \rightarrow \infty} d\left(x_{n}, p\right)$ exists and $0<a \leq \lambda_{n}^{(i)} \leq b<1$ for all $i=0,1, \ldots, N$, we get that

$$
\lim _{n \rightarrow \infty} d\left(x_{n}, T x_{n}\right)=0 \quad \text { and } \quad \lim _{n \rightarrow \infty} d\left(W_{n}^{(k-1)}, T^{k} x_{n}\right)=0 \quad \text { for all } k=2,3, \ldots, N .
$$

For $k=2,3, \ldots, N$, we have

$$
\begin{aligned}
d\left(x_{n}, T^{k} x_{n}\right) & \leq d\left(x_{n}, W_{n}^{(k-1)}\right)+d\left(W_{n}^{(k-1)}, T^{k} x_{n}\right) \\
& =d\left(x_{n}, \bigoplus_{i=0}^{k-1} \frac{\lambda_{n}^{(i)}}{\sum_{j=0}^{k-1} \lambda_{n}^{(j)}} T^{i} x_{n}\right)+d\left(W_{n}^{(k-1)}, T^{k} x_{n}\right) \\
& \leq \sum_{i=0}^{k-1} \frac{\lambda_{n}^{(i)}}{\sum_{j=0}^{k-1} \lambda_{n}^{(j)}} d\left(x_{n}, T^{i} x_{n}\right)+d\left(W_{n}^{(k-1)}, T^{k} x_{n}\right) \\
& =\sum_{i=1}^{k-1} \frac{\lambda_{n}^{(i)}}{\sum_{j=0}^{k-1} \lambda_{n}^{(j)}} d\left(x_{n}, T^{i} x_{n}\right)+d\left(W_{n}^{(k-1)}, T^{k} x_{n}\right) .
\end{aligned}
$$

This implies by (3.4) that

$$
\lim _{n \rightarrow \infty} d\left(x_{n}, T^{k} x_{n}\right)=0 \quad \text { for all } k=1,2, \ldots, N .
$$

We now let $\omega_{\Delta}\left(x_{n}\right):=\bigcup A\left(C,\left\{u_{n}\right\}\right)$, where the union is taken over all subsequences $\left\{u_{n}\right\}$ of $\left\{x_{n}\right\}$. We claim that $\omega_{\Delta}\left(x_{n}\right) \subset F(T)$. Let $u \in \omega_{\Delta}\left(x_{n}\right)$. Then there exists a subsequence $\left\{u_{n}\right\}$ of $\left\{x_{n}\right\}$ such that $A\left(C,\left\{u_{n}\right\}\right)=\{u\}$. By Lemma 1.5, there exists a subsequence $\left\{u_{n_{k}}\right\}$ of $\left\{u_{n}\right\}$ such that $\Delta-\lim _{k \rightarrow \infty} u_{n_{k}}=y \in C$. It implies by (3.5) and Lemma 3.5 that $y \in F(T)$. Then, $\lim _{n \rightarrow \infty} d\left(x_{n}, y\right)$ exists. Suppose that $u \neq y$. By the uniqueness of asymptotic centers, we get

$$
\begin{aligned}
\limsup _{k \rightarrow \infty} d\left(u_{n_{k}}, y\right) & <\limsup _{k \rightarrow \infty} d\left(u_{n_{k}}, u\right) \\
& \leq \limsup _{n \rightarrow \infty} d\left(u_{n}, u\right)
\end{aligned}
$$




$$
\begin{aligned}
& <\limsup _{n \rightarrow \infty} d\left(u_{n}, y\right) \\
& =\limsup _{n \rightarrow \infty} d\left(x_{n}, y\right) \\
& =\limsup _{k \rightarrow \infty} d\left(u_{n_{k}}, y\right) .
\end{aligned}
$$

This is a contradiction, hence $u=y \in F(T)$. This shows that $\omega_{\Delta}\left(x_{n}\right) \subset F(T)$.

Next, we show that $\omega_{\Delta}\left(x_{n}\right)$ consists of exactly one point. Let $\left\{u_{n}\right\}$ be a subsequence of $\left\{x_{n}\right\}$ with $A\left(C,\left\{u_{n}\right\}\right)=\{u\}$ and let $A\left(C,\left\{x_{n}\right\}\right)=\{z\}$. Since $u \in \omega_{\Delta}\left(x_{n}\right) \subset F(T)$, it follows that $\lim _{n \rightarrow \infty} d\left(x_{n}, u\right)$ exists. We will show that $z=u$. To show this, suppose not. By the uniqueness of asymptotic centers, we get

$$
\begin{aligned}
\limsup _{n \rightarrow \infty} d\left(u_{n}, u\right) & <\limsup _{n \rightarrow \infty} d\left(u_{n}, z\right) \\
& \leq \limsup _{n \rightarrow \infty} d\left(x_{n}, z\right) \\
& <\limsup _{n \rightarrow \infty} d\left(x_{n}, u\right) \\
& =\limsup _{n \rightarrow \infty} d\left(u_{n}, u\right),
\end{aligned}
$$

which is a contradiction, and so $z=u$. Hence, $\left\{x_{n}\right\} \Delta$-converges to a fixed point $u$ of $T$. Since $F(T)$ is a closed convex subset of $X$ and $d\left(x_{n+1}, p\right) \leq d\left(x_{n}, p\right)$ for all $p \in F(T)$ and $n \in \mathbb{N}$, we obtain by Lemma 1.6 that $\left\{\pi x_{n}\right\}$ converges strongly to some element in $F(T)$, say $q$. Thus, by the property of $\pi$, we obtain that

$$
\begin{aligned}
\limsup _{n \rightarrow \infty} d\left(x_{n}, q\right) & \leq \limsup _{n \rightarrow \infty}\left(d\left(x_{n}, \pi x_{n}\right)+d\left(\pi x_{n}, q\right)\right) \\
& =\limsup _{n \rightarrow \infty} d\left(x_{n}, \pi x_{n}\right) \\
& \leq \limsup _{n \rightarrow \infty} d\left(x_{n}, u\right) .
\end{aligned}
$$

This implies, by the uniqueness of asymptotic centers, that $q=u$. This means $u=$ $\lim _{n \rightarrow \infty} \pi x_{n}$.

Taking $N=2$ in Theorem 3.7, we obtain the following $\Delta$-convergence theorem of a 2-generalized hybrid mapping in $\mathrm{CAT}(0)$ spaces.

Theorem 3.8 Let $C$ be a nonempty closed and convex subset of a complete CAT( 0$)$ space $X$. Let $T: C \rightarrow C$ be a 2-generalized hybrid mapping, i.e., there are $\alpha_{1}, \alpha_{2}, \beta_{1}, \beta_{2} \in \mathbb{R}$ such that

$$
\begin{aligned}
& \alpha_{1} d\left(T^{2} x, T y\right)^{2}+\alpha_{2} d(T x, T y)^{2}+\left(1-\alpha_{1}-\alpha_{2}\right) d(x, T y)^{2} \\
& \leq \beta_{1} d\left(T^{2} x, y\right)^{2}+\beta_{2} d(T x, y)^{2}+\left(1-\beta_{1}-\beta_{2}\right) d(x, y)^{2}
\end{aligned}
$$

for all $x, y \in C$. Assume that $F(T) \neq \emptyset$ and $\alpha_{1}+\alpha_{2} \in(-\infty, 0] \cup[1, \infty)$ and $\beta_{1}+\beta_{2} \in[0, \infty)$. Let $\pi: C \rightarrow F(T)$ be the nearest point projection mapping. For $x_{1} \in C$, let $\left\{x_{n}\right\}$ be a sequence defined by

$$
x_{n+1}=\bigoplus_{i=0}^{2} \lambda_{n}^{(i)} T^{i} x_{n} \quad \text { for all } n \in \mathbb{N}
$$


where $\left\{\lambda_{n}^{(i)}\right\}$ is a sequence in $[0,1]$ with $0<a \leq \lambda_{n}^{(i)} \leq b<1$ for all $i=0,1,2$ and $\sum_{i=0}^{2} \lambda_{n}^{(i)}=1$. Then $\left\{x_{n}\right\} \Delta$-converges to a fixed point $u$ of $T$, where $u=\lim _{n \rightarrow \infty} \pi x_{n}$.

Taking $N=1$ in Theorem 3.7, we obtain the following $\Delta$-convergence theorem of a generalized hybrid mapping in $\mathrm{CAT}(0)$ spaces.

Theorem 3.9 Let $C$ be a nonempty closed and convex subset of a complete CAT(0) space X. Let $T: C \rightarrow C$ be a generalized hybrid mapping, i.e., there are $\alpha, \beta \in \mathbb{R}$ such that

$\alpha d(T x, T y)^{2}+(1-\alpha) d(x, T y)^{2} \leq \beta d(T x, y)^{2}+(1-\beta) d(x, y)^{2}$

for all $x, y \in C$. Assume that $F(T) \neq \emptyset$ and $\alpha \in(-\infty, 0] \cup[1, \infty)$ and $\beta \in[0, \infty)$. Let $\pi: C \rightarrow$ $F(T)$ be the nearest point projection mapping. For $x_{1} \in C$, let $\left\{x_{n}\right\}$ be a sequence defined by

$$
x_{n+1}=\lambda_{n}^{(0)} x_{n} \oplus \lambda_{n}^{(1)} T x_{n} \quad \text { for all } n \in \mathbb{N},
$$

where $\left\{\lambda_{n}^{(0)}\right\}$ and $\left\{\lambda_{n}^{(1)}\right\}$ are sequences in $[0,1]$ with $0<a \leq \lambda_{n}^{(0)}, \lambda_{n}^{(1)} \leq b<1$ and $\lambda_{n}^{(0)}+\lambda_{n}^{(1)}=1$. Then $\left\{x_{n}\right\} \Delta$-converges to a fixed point $u$ of $T$, where $u=\lim _{n \rightarrow \infty} \pi x_{n}$.

\section{Competing interests}

The author declares that he has no competing interests.

\section{Acknowledgements}

The author would like to thank the referees for valuable suggestions on the paper.

Received: 22 March 2013 Accepted: 4 July 2013 Published: 18 July 2013

\section{References}

1. Takahashi, W: A convexity in metric space and nonexpansive mappings. Kodai Math. Semin. Rep. 22, 142-149 (1970)

2. Aoyama, K, Eshita, K, Takahashi, W: Iteration processes for nonexpansive mappings in convex metric spaces. In: Proceedings of the International Conference on Nonlinear and Convex Analysis, pp. 31-39 (2005)

3. Shimizu, T, Takahashi, W: Fixed points of multivalued mappings in certain convex metric spaces. Topol. Methods Nonlinear Anal. 8, 197-203 (1996)

4. Goebel, K, Kirk, WA: Topics in Metric Fixed Point Theory. Cambridge University Press, Cambridge (1990)

5. Goebel, K, Reich, S: Uniform Convexity, Hyperbolic Geometry, and Nonexpansive Mappings. Monographs and Textbooks in Pure and Applied Mathematics, vol. 83. Dekker, New York (1984)

6. Dhompongsa, S, Kirk, WA, Sims, B: Fixed points of uniformly Lipschitzian mappings. Nonlinear Anal. 65, 762-772 (2006)

7. Phuengrattana, W, Suantai, S: Existence theorems for generalized asymptotically nonexpansive mappings in uniformly convex metric spaces. J. Convex Anal. 20, 753-761 (2013)

8. Bridson, M, Haefliger, A: Metric Spaces of Non-Positive Curvature. Springer, Berlin (1999)

9. Kirk, WA: Geodesic geometry and fixed point theory II. In: International Conference on Fixed Point Theory and Applications, pp. 113-142. Yokohama Publishers, Yokohama (2004)

10. Kirk, WA: Geodesic geometry and fixed point theory. In: Seminar of Mathematical Analysis, Malaga/Seville (2002/2003). Colecc. Abierta, vol. 64, pp. 195-225. Univ. Sevilla Secr. Publ., Seville (2003)

11. Dhompongsa, S, Kaewkhao, A, Panyanak, B: Lim's theorems for multivalued mappings in CAT(0) spaces. J. Math. Anal. Appl. 312, 478-487 (2005)

12. Dhompongsa, S, Kaewkhao, A, Panyanak, B: On Kirk's strong convergence theorem for multivalued nonexpansive mappings on CAT(0) spaces. Nonlinear Anal. 75, 459-468 (2012)

13. Chaoha, P, Phon-on, A: A note on fixed point sets in CAT(0) spaces. J. Math. Anal. Appl. 320, $983-987$ (2006)

14. Leuştean, L: A quadratic rate of asymptotic regularity for CAT(0)-spaces. J. Math. Anal. Appl. 325, 386-399 (2007)

15. Kirk, WA, Panyanak, B: A concept of convergence in geodesic spaces. Nonlinear Anal. 68, 3689-3696 (2008)

16. Kirk, WA: Fixed point theorems in CAT(0) spaces and $\mathbb{R}$-trees. Fixed Point Theory Appl. 2004, 309-316 (2004)

17. Lin, LJ, Chuang, CS, Yu, ZT: Fixed point theorems and $\Delta$-convergence theorems for generalized hybrid mappings on CAT(0) spaces. Fixed Point Theory Appl. 2011, 49 (2011)

18. Nanjaras, B, Panyanak, B, Phuengrattana, W: Fixed point theorems and convergence theorems for Suzuki-generalized nonexpansive mappings in CAT(0) spaces. Nonlinear Anal. Hybrid Syst. 4, 25-31 (2010)

19. Phuengrattana, W: Approximating fixed points of Suzuki-generalized nonexpansive mappings. Nonlinear Anal. Hybrid Syst. 5, 583-590 (2011) 
20. Phuengrattana, W, Suantai, S: Strong convergence theorems for a countable family of nonexpansive mappings in convex metric spaces. Abstr. Appl. Anal. 2011, Article ID 929037 (2011)

21. Cho, YJ, Ćirić, L, Wang, SH: Convergence theorems for nonexpansive semigroups in CAT(0) spaces. Nonlinear Anal. 74, 6050-6059 (2011)

22. Kirk, WA: Fixed point theorems in CAT(0) spaces and $\mathbb{R}$-trees. Fixed Point Theory Appl. 2004, 309-316 (2004)

23. Saejung, S: Halpern's iteration in CAT(0) spaces. Fixed Point Theory Appl. 2010, Article ID 471781 (2010)

24. Fukhar-ud-din, H, Khan, AR, Akhtar, Z: Fixed point results for generalized nonexpansive maps in uniformly convex metric spaces. Nonlinear Anal. 75, 4747-4760 (2012)

25. Khan, AR, Khamsi, MA, Fukhar-ud-din, H: Strong convergence of a general iteration scheme in CAT(0) spaces. Nonlinear Anal. 74, 783-791 (2011)

26. Khan, AR, Fukhar-ud-din, H, Domlo, AA: Approximating fixed points of some maps in uniformly convex metric spaces Fixed Point Theory Appl. 2010, Article ID 385986 (2010)

27. Fukhar-ud-din, H, Domlo, AA, Khan, AR: Strong convergence of an implicit algorithm in CAT(0) spaces. Fixed Point Theory Appl. 2011, Article ID 173621 (2011)

28. Bruhat, F, Tits, J: Groupes réductifs sur un corps local. Publ. Math. Inst. Hautes Études Sci. 41, 5-251 (1972)

29. Dhompongsa, S, Panyanak, B: On $\Delta$-convergence theorems in CAT(0) spaces. Comput. Math. Appl. 56, 2572-2579 (2008)

30. Lim, TC: Remark on some fixed point theorems. Proc. Am. Math. Soc. 60, 179-182 (1976)

31. Takahashi, W, Toyoda, M: Weak convergence theorems for nonexpansive mappings and monotone mappings. J. Optim. Theory Appl. 118, 417-428 (2003)

32. Kocourek, P, Takahashi, W, Yao, JC: Fixed point theorems and weak convergence theorems for generalized hybrid mappings in Hilbert spaces. Taiwan. J. Math. 14, 2497-2511 (2010)

33. Takahashi, W, Yao, JC: Weak convergence theorems for generalized hybrid mappings in Banach spaces. J. Nonlinear Anal. Optim. 2(1), 147-158 (2011)

34. Maruyama, T, Takahashi, W, Yao, M: Fixed point and mean ergodic theorems for new nonlinear mappings in Hilbert spaces. J. Nonlinear Convex Anal. 12, 185-197 (2011)

35. Hojo, M, Takahashi, W, Termwuttipong, I: Strong convergence theorems for 2-generalized hybrid mappings in Hilbert spaces. Nonlinear Anal. 75, 2166-2176 (2012)

36. Aoyama, K, lemoto, S, Kohsaka, F, Takahashi, W: Fixed point and ergodic theorems for $\boldsymbol{\lambda}$-hybrid mappings in Hilbert spaces. J. Nonlinear Convex Anal. 11, 335-343 (2010)

37. Kohsaka, F, Takahashi, W: Fixed point theorems for a class of nonlinear mappings related to maximal monotone operators in Banach spaces. Arch. Math. 91, 166-177 (2008)

38. Takahashi, W: Fixed point theorems for new nonlinear mappings in a Hilbert space. J. Nonlinear Convex Anal. 11 , 79-88 (2010)

39. Takahashi, W: Nonlinear Function Analysis. Yokahama Publishers, Yokahama (2000)

40. Sturm, KT: Probability measures on metric spaces of nonpositive curvature. In: Heat Kernels and Analysis on Manifolds, Graphs and Metric Spaces (Paris, 2002). Contemp. Math., vol. 338, pp. 357-390. Am. Math. Soc., Providence (2003)

doi:10.1186/1687-1812-2013-188

Cite this article as: Phuengrattana: Fixed point theorems for $\mathrm{N}$-generalized hybrid mappings in uniformly convex metric spaces. Fixed Point Theory and Applications 2013 2013:188.

\section{Submit your manuscript to a SpringerOpen ${ }^{\odot}$ journal and benefit from:}

- Convenient online submission

- Rigorous peer review

- Immediate publication on acceptance

- Open access: articles freely available online

- High visibility within the field

- Retaining the copyright to your article 